\title{
STRATEGIES FOR THE USE OF NON-STATIN THERAPIES
}

\author{
Angela Pirillo ${ }^{1,2}$, Giuseppe Danilo Norata ${ }^{1,3}$, Alberico Luigi Catapano ${ }^{1,4}$
}

${ }^{1}$ Center for the Study of Atherosclerosis, E. Bassini Hospital, Cinisello Balsamo, Milan, Italy.

2IRCCS Multimedica Hospital, Sesto San Giovanni, Milan, Italy.

${ }^{3}$ Department of Pharmacological and Biomolecular Sciences, Università degli Studi di Milano, Milan, Italy.

${ }^{4}$ School of Biomedical Sciences, Curtin Health Innovation Research Institute, Curtin University, Perth, Western Australia.

\section{Corresponding Author:}

Prof. Alberico L. Catapano

Department of Pharmacological and Biomolecular Sciences, University of Milan and IRCCS Multimedica

Via Balzaretti, 9 - 20133 Milan ITALY

Phone number: +39250318302

Fax number: +39250318386

e-mail: alberico.catapano@unimi.it 


\section{Abstract}

\section{Purpose of review}

Dyslipidaemias are a major risk factor for cardiovascular disease (CVD); in particular high levels of low density lipoprotein cholesterol (LDL-C) have been associated to a higher cardiovascular risk. Reducing LDL-C levels decreases the risk of coronary heart disease (CHD), and the greater the LDL-C reduction, the greater the cardiovascular risk decrease. Although statins represent the first line lipid-lowering therapy, many patients do not reach the recommended goals or exhibit adverse side effects leading to therapy discontinuation; in addition, a significant percentage of statintreated patients continue to experience cardiovascular events even in the presence of well controlled LDL-C levels, due to alterations in other lipid/lipoprotein classes, including triglycerides and high-density lipoprotein cholesterol.

\section{Recent findings}

These conditions require further therapeutic interventions to achieve the recommended lipid goals. Several drugs have been developed to address these needs. Recent studies have shown that the association of ezetimibe with rosuvastatin or atorvastatin results in a better hypolipidaemic effect; beside this, PCSK9 inhibitors significantly reduce LDL-C levels and cardiovascular events.

\section{Summary}

For patients intolerant to statins or not able to reach the recommended LDL-C levels despite maximal tolerated dose of statin, or exhibiting additional lipid alterations, several drugs are available that can be used either in monotherapy or on top of the maximally tolerated dose of statins. 
Keywords: dyslipidaemias; hypercholesterolaemia; LDL-C; triglycerides; statin-intolerance; ezetimibe; PCSK9 inhibitors 


\section{Introduction}

Dyslipidaemias, and in particular hypercholesterolaemia, represent a major risk factor for cardiovascular disease (CVD). Evidence from clinical trials, epidemiological and Mendelian randomization studies has established the causality for low density lipoprotein cholesterol (LDLC) and/or triglyceride rich lipoproteins (TGRL) in atherosclerotic cardiovascular disease [1-3]. Data from meta-analyses of statin trials further proved that reducing LDL-C levels reduces the risk of coronary heart disease (CHD), and that the greater the LDL-C reduction, the greater the cardiovascular (CV) risk decrease [4-7].

Despite the established clinical efficacy of statin therapy, several statin-treated patients continue to experience cardiovascular events, even in the presence of well controlled LDL-C levels [8]. This observation suggests that an alteration in the plasma levels of other lipids (such as high triglycerides and/or low HDL-C plasma levels) may confer a residual risk $[9,10]$. This may be the case of diabetic patients, who are characterized by the atherogenic dyslipidaemia (low HDL-C levels, high TG and small dense LDL levels) [11]. A causal association between elevated TG, TGrich lipoproteins or their remnants and increased CVD risk has also been established, independent of LDL-C or HDL-C levels [3, 12-17]. Statins may affect TG and HDL-C levels: a dose-dependent TG reduction has been reported, ranging from 9 up to $25 \%$, with greater reductions observed in patients with higher baseline TG values $[18,19]$; in addition, all statins are able to increase HDL-C and apoA-I independently of LDL-C reduction but related to the baseline TG levels [20, 21].

Thus, statins might represent a reasonable approach also for the treatment of subjects with increased TG levels and increased CV risk. Many patients, however, do not reach the desired goal despite maximal tolerated statin therapy or experience side effects, particularly muscle-related adverse events, when taking statins. The incidence of statin-related side effects is further increased in subjects undergoing polytherapy, which increases the probability of drug-drug interactions and 
the appearance of adverse side-effects [22]. This may lead to therapy discontinuation and makes difficult the management of patients intolerant to statins, particularly those at high or very high CV risk, for whom high doses of high intensity statins are recommended.

Therefore, although statin therapy represents the first approach in the management of dyslipidaemias, there are patients that require further therapeutic interventions to achieve the recommended lipid goals or patients where statins are less effective. This review will focus on three categories of patients that include 1) statin-intolerant patients, 2) patients not at target and 3) patients with marked hypertriglyceridaemia (HTG).

\section{1) Patients with statin-intolerance}

Statin intolerance is defined as the inability to tolerate statins, independently on the type and dose [23]. Despite a possible causal association between statin use and myopathy, randomised controlled trials have suggested a small impact of statin therapy on less severe muscle pain (i.e., myalgia) or weakness [24]. In the clinical practice, such symptoms are commonly attributed to statins, as also suggested by the so-called nocebo effect, with an increased rate of muscle-related effects only after having been informed of the statin treatment compared with the blinded use [25]. The occurrence of statin-related side effects, which appears to be dose-dependent, may lead to therapy discontinuation, and therefore increases the CV risk of these patients. Thus, the availability of alternative non-statin lipid-lowering drugs (Figure 1), which include bile acid sequestrants, ezetimibe or PCSK9 inhibitors that may be used either as monotherapy or in combination with lower doses of statins (thus reducing the chance of statin-induced side effects), is essential to reduce the CV risk of statin-intolerant patients. 
Specifically, bile acid sequestrants (cholestyramine, colestipol and more recently colesevelam) act by binding bile acids thus limiting their reabsorption; indirectly, this results in an increased expression of hepatic LDL receptor (LDLR) and increased LDL catabolism. These drugs reduce LDL-C by $18-25 \%$, with an additional $10-20 \%$ reduction when added to a statin [26]. This effect translates into a reduction of $\mathrm{CV}$ events which is proportional to the degree of LDL-C lowering achieved $[27,28]$. Bile acid sequestrants may be used also in patients intolerant to statins as well as in those who do not tolerate increasing dose of statins, and may be combined with other lipidlowering drugs such as ezetimibe, resulting in a greater reduction of LDL-C levels compared with ezetimibe alone [29].

Ezetimibe reduces intestinal uptake of dietary and biliary cholesterol by inhibiting the interaction with the Niemann-Pick C1-like protein 1 (NPC1L1) [30]. Consequently, the delivery of diet cholesterol to the liver is reduced, thus favouring the increase of hepatic LDLR expression and reducing LDL-C plasma levels. The validity of this approach is confirmed by the fact that NPC1L1 inactivating mutations are associated with lower plasma LDL-C levels and reduced CV risk [31]. A pooled analysis of over 21,000 subjects from 27 clinical trials showed that combining ezetimibe with a statin induces a greater lipid-lowering effect than statin monotherapy [32]. The administration of ezetimibe in combination with low dose of simvastatin (10 mg) for 6 months significantly reduces LDL-C levels and a high percentage of patients achieves the therapeutic target; more importantly, no adverse events were reported during the study [33]. The effectiveness of this combination in reducing CV events has been confirmed by several clinical trials [34-36]. Additional benefits are achieved when ezetimibe is combined with atorvastatin or rosuvastatin [37-40]. Ezetimibe may also be combined with bile acid sequestrants, resulting in an additional reduction of LDL-C levels [29]. 
Proprotein convertase subtilisin kexin 9 (PCSK9) is a protein involved in the control of hepatic LDLR expression [41]; upon binding to the LDLR, it favours its targeting to the lysosome for degradation, thus resulting in a reduced LDLR expression and increased LDL-C levels. Reduced levels or function of this protein are associated with hypocholesterolaemia and reduced CV risk, suggesting it as a pharmacological target to reduce hypercholesterolaemia [42]. Two fully human monoclonal antibodies against PCSK9, alirocumab and evolocumab, which decrease LDL-C by up to $70 \%$, have been approved for the treatment of patients with primary hypercholesterolaemia not at LDL-C goal despite maximal tolerated LLT as well as for statin-intolerant patients. A number of randomized clinical trials have evaluated the effect of evolocumab in statin-intolerant patients [4345]. The GAUSS-3 study was designed as a two-phase trial to first identify patients with musclerelated adverse events during an atorvastatin rechallenge phase to proceed to the second phase of treatment with evolocumab or ezetimibe [45]. A greater reduction of LDL-C levels was reported in evolocumab-treated patients compared with ezetimibe after 24 weeks (treatment difference vs. ezetimibe $\sim-36 \%$ ), with a good tolerability of both drugs [45]. Similar results were observed when alirocumab was compared to ezetimibe in statin-intolerant patients: the reduction of LDL-C levels was $-45 \%$ with alirocumab and $-14.6 \%$ with ezetimibe (mean treatment difference $-30.4 \%$ ); the incidence of muscle-related adverse events were lower when patients had evolocumab compared with the rechallenge phase with atorvastatin [46]. These data indicate a higher effectiveness of antiPCSK9 mAbs in reducing LDL-C levels compared with ezetimibe in statin-intolerant hypercholesterolaemic patients. Furthermore, the recent data from the FOURIER trial show that inhibition of PCSK9 effectively reduces LDL-C levels and significantly reduces the risk of cardiovascular events [47], suggesting that this therapeutic approach could be effective in reducing the CV risk of patients intolerant to statins. 


\section{2) Hypercholesterolaemic patients not at target despite maximized statin therapy}

Despite the established efficacy of statin therapy in reducing LDL-C levels and CV events, a considerable proportion of patients do not achieve the LDL-C levels recommended by guidelines based on their CV risk [48]. There are specific groups of patients in whom this proportion is significantly higher, such as patients with familial hypercholesterolaemia (FH) or patients with established CVD or very high CV risk, thus indicating the need of additional lipid-lowering therapies (Figure 1).

Due to the lifelong exposure to high levels of cholesterol, FH patients are at high CV risk; although statin therapy represents always the first approach also in these patients, the effectiveness of statins is strictly related to the presence of a functional LDLR, due to the mechanism of action of this class of drugs. Thus, FH patients on statin therapy are often far from the recommended LDL-C levels [49] and need to be treated with lipid-lowering drugs with a mechanism of action differing from that of statins. The addition of ezetimibe to a statin in $\mathrm{FH}$ patients results in an additional 1015\% LDL-C reduction [50-52], which may translate into a further reduction of CV risk. Heterozygous FH patients are the principle candidates for the therapy with anti-PCSK9 mAbs. Several clinical trials have in fact reported a robust benefit in $\mathrm{HeFH}$ patients following the addition of an anti-PCSK9 $\mathrm{mAb}$ to their current lipid-lowering therapy (maximally tolerated statin with/without other lipid-lowering therapy) [53-59]. Both alirocumab and evolocumab significantly decrease LDL-C levels by $-42.5 \%$ up to $-67.9 \%$ in $\mathrm{HeFH}$ under different clinical settings, with higher proportion of patients under anti-PCSK9 mAb therapy reaching the recommended LDL-C levels based on their CV risk [53-59]. The LDL-C lowering effect persists over time and after 78 weeks the LDL-C levels are still significantly low; similarly, the proportion of patients reaching their goal is maintained up to 78 weeks [60]. A pooled analysis of safety data for alirocumab in HeFH patients showed that rates of treatment-emergent adverse events (TEAEs) as well as TEAEs 
leading to discontinuation are similar in alirocumab and placebo groups [60]. These findings suggest that PCSK9 inhibitors represent a major opportunity for these difficult-to-treat patients to reach the recommended LDL-C levels and, as a consequence, to reduce their high CV risk.

Patients with established CVD may benefit from the addition of ezetimibe to a statin. As reported by the IMPROVE-IT trial, the addition of ezetimibe to simvastatin not only results in a greater reduction of LDL-C levels compared with simvastatin monotherapy, but also reduces the incidence of cardiovascular events in patients with a recent acute coronary syndrome [34]. The absolute benefit deriving from the combination therapy is small, but significant, thus confirming that lower LDL-C levels always associate with lower CV risk. Ezetimibe thus represents a second line of therapy that can be added to the current statin therapy to achieve the therapeutic goal; this may be of special relevance in patients at high CV risk who cannot reach the LDL-C target with the maximal tolerated dose of statins [48]. The FOURIER trial showed that evolocumab added to a statin therapy significantly reduced LDL-C levels (mean percentage reduction $59 \%$ as compared with placebo) and reduced the risk of cardiovascular events (hazard ratio, 0.85; $\mathrm{p}<0.001$ ) in patients with atherosclerotic cardiovascular disease and LDL-C >70 mg/dL (1.81 mmol/L) [47]. A secondary analysis of the FOURIER trial showed that patients who achieved progressively lower LDL-C levels at 4 weeks exhibited progressively a lower rate of cardiovascular events, with adjusted hazard ratios of 0.69 for the primary endpoint and 0.59 for key secondary endpoints in the group with LDL-C $<10 \mathrm{mg} / \mathrm{dL}$ or $0.26 \mathrm{mmol} / \mathrm{L}$ at 4 weeks [61]; no serious adverse events or adverse events leading to drug discontinuation were observed in patients achieving such very low LDL-C levels, thus suggesting that lower LDL-C levels $(<0.5 \mathrm{mmol} / \mathrm{L})$ can be safely considered for high risk patients. In addition, evolocumab added to a moderate or high-intensity statin therapy induced atherosclerotic plaque regression compared with placebo in patients with angiographic coronary disease [62]. Similarly, alirocumab significantly reduced LDL-C plasma levels compared 
with either placebo (difference $-45.9 \%$ ) or ezetimibe (difference: $-29.8 \%$ ) in high risk patients on maximally tolerated doses of statins; accordingly, a higher proportion of patients on alirocumab achieved the recommended LDL-C levels [63, 64].

\section{3) Patients with hypertriglyceridaemia}

For the purpose of this review, we discuss exclusively data about patients with mild-to-moderate hypertriglyceridaemia $(150-880 \mathrm{mg} / \mathrm{dL}, 1.7-10 \mathrm{mmol} / \mathrm{L})$; we did not include patients with severe hypertriglyceridaemia (>880 mg/dL, $>10 \mathrm{mmol} / \mathrm{L})$, typically associated with a high risk of pancreatitis and determined by monogenic mutations, who require a different therapeutic approach [15].

Mendelian randomization studies have reported a correlation between both high non-fasting TG levels and remnant cholesterol (i.e. the cholesterol of TG-rich lipoproteins) and increased risk of CV events and all-cause mortality [16, 17]. Despite this, the final evidence of benefits of lowering TG levels is still lacking. To date, no large clinical trials addressing specific clinical outcomes have been completed in which subjects were recruited based on their elevated levels of TG or TG-rich lipoproteins, and thus, the evidence to support TG-lowering therapies is less robust than for LDLC. Thus, although clinical trials investigating the effects of TG-lowering drugs on cardiovascular outcomes have reported conflicting results, a recent meta-analysis reported an overall modest CV risk reduction $(12 \%)$, which however was more consistent in specific subject subgroups, such as those with high TG (18\% risk reduction) and even more in subjects with high TG and low HDL-C (29\% risk reduction) [65].

Beside these considerations, it is worth noting that many patients treated with statins still exhibit high levels of non-HDL-C, which reflects an increase in TG-rich lipoprotein cholesterol [66, 67], suggesting the need of additional therapies able to lower specifically TG levels. The available 
pharmacological interventions that substantially reduce TG and TG-rich lipoprotein cholesterol include fibrates and n-3 PUFAs.

Fibrates are agonists of peroxisome proliferator-activated receptor- $\alpha$ (PPAR- $\alpha$ ), which act by regulating various steps in lipid and lipoprotein metabolism, resulting in reduced fasting TG levels, post-prandial TG and TG-rich lipoprotein remnant particles. Contrasting results have been reported from meta-analyses of fibrates clinical trials; in fact, while some meta-analyses did not observe any cardiovascular benefit $[68,69]$, others reported a reduction of CV events in specific group of patients with high TG levels associated with low HDL-C [70-72]. Thus, although overall these results may be suggestive of a clinical benefit in subgroups of high CV risk patients, specific clinical trials are essential to substantiate this hypothesis. The novel selective PPAR $\alpha$ modulator pemafibrate is currently under investigation in diabetic patients to evaluate the effect of TGlowering on cardiovascular outcomes (PROMINENT, NCT03071692). N-3 fatty acids [eicosapentaenoic acid (EPA) and docosahexaenoic acid (DHA) efficiently reduce TG levels by several mechanisms [73]. The recommended doses of EPA+DHA is 2-4 g/day, which translate into a $25-30 \%$ reduction of TG levels [73]. Whether this relevant reduction of plasma TG levels may also translate into a clinical benefit is still debated. In fact, although some studies reported significant reduction of CV outcomes following the treatment with omega-3 fatty acids [74, 75], other studies failed to observe any kind of protection [76]. A large systematic review including data from 20 clinical trials reported that omega-3 fatty acids protected against vascular death $(R R=0.96, P=0.03)$ but overall there was no effect on composite cardiovascular events coronary events ( $R R=0.96$, $\mathrm{P}=0.24)$ [77]. Two ongoing randomized placebo-controlled trials (REDUCE-IT, NCT01492361 and STRENGTH, NCT02104817) are evaluating the effect of omega-3 fatty acids on CVD outcomes in subjects with high TG levels. 


\section{CONCLUSIONS}

The reduction of LDL cholesterol is one of the pillars in the prevention of CVD. Statins are the first line therapy as suggested by the major guidelines and the benefit is directly proportional to the absolute LDL-C level decrease and the absolute CV risk of the patient. Yet there are circumstances under which either the hypolipidaemic response is deemed as insufficient (especially in patients with the so-called statin intolerance) or patients are affected of other forms of dyslipidaemia that confer additional CV risk. The size of these populations varies widely from survey to survey but certainly account for a relatively large part of the high and very high risk population and other non statin treatment represent a powerful tool in the armamentarium of the physician to provide the best $\mathrm{CV}$ protection possible. 


\section{KEY POINTS}

- Despite the clinical efficacy of statin therapy, several patients still need further drugs to reduce their $\mathrm{CV}$ risk

- Statin-intolerant patients require alternative non-statin therapies to manage their CV risk, which may include bile acid sequestrants, ezetimibe in combination with low doses of statins, and PCSK9 inhibitors, which seem to represent a valid approach to reduce LDL-C levels and CV risk

- Specific groups of patients such as those with FH or with established cardiovascular disease, who are at high $\mathrm{CV}$ risk, often require additional drugs for the management of their CV risk, which may include PCSK9 inhibitors or ezetimibe in association with a statin

- Patients with severe HTG have an increased CV risk, and may be treated with fibrates or omega-3 fatty acids, although the final proof of their ability to reduce the CV risk in these patients is still debated.

- Here we discuss the recent findings on newest hypolipidaemic drugs as well as findings from drugs previously used for the management of these specific groups of patients 


\section{Acknowledgments}

None

Financial support and sponsorship

None

\section{Conflict of interest}

A.P. reports no disclosures. G.D.N received research funding and/or honoraria for advisory boards, consultancy or speaker bureau from Aegerion, Amgen, Pfizer, Recordati, SanofiRegeneron. A.L.C. received research funding and/or honoraria for advisory boards, consultancy or speaker bureau from Aegerion, Amgen, AstraZeneca, Eli Lilly, Genzyme, Mediolanum, Merck or MSD, Pfizer, Recordati, Rottapharm, Sanofi-Regeneron, Sigma-Tau. 


\section{Legend to the figure}

Figure 1. Sites of action of statins and other lipid-lowering drugs

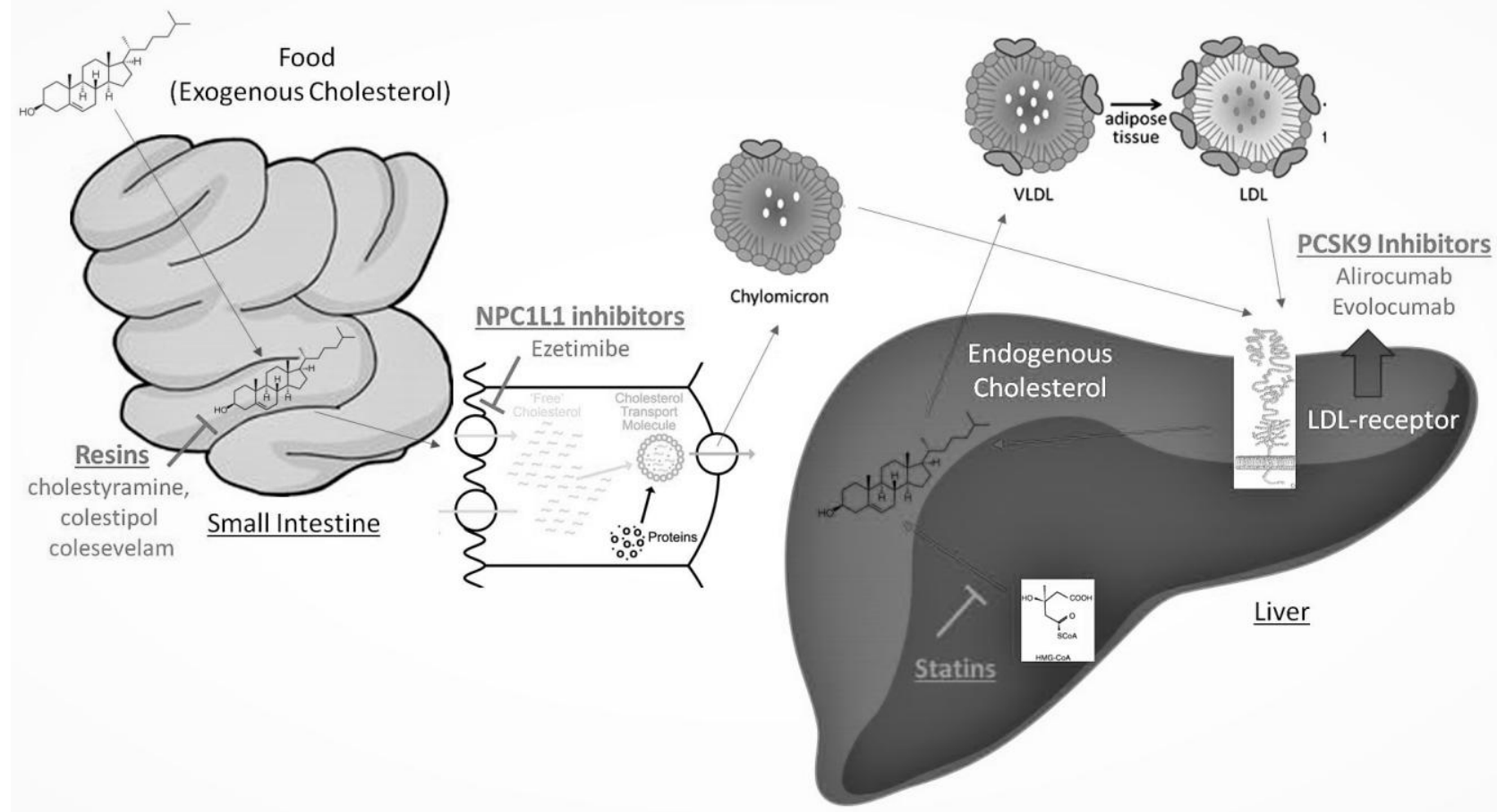




\section{References}

[1] Ference BA, Ginsberg H, Graham I et al. Low-density lipoproteins cause atherosclerotic cardiovascular disease. 1. Evidence from genetic, epidemiologic and clinical studies. A Consensus Statement from the European Atherosclerosis Society Consensus Panel. Eur Heart J 2017; 10.1093/eurheartj/ehx144.

**An overall evaluation of clinical and genetic evidence establishing that LDL unequivocally cause atherosclerotic disease

[2] Crosby J, Peloso GM, Auer PL et al. Loss-of-function mutations in APOC3, triglycerides, and coronary disease. N Engl J Med 2014; 371:22-31.

[3] Do R, Willer CJ, Schmidt EM et al. Common variants associated with plasma triglycerides and risk for coronary artery disease. Nat Genet 2013; 45:1345-1352.

[4] Baigent C, Blackwell L, Emberson J et al. Efficacy and safety of more intensive lowering of LDL cholesterol: a meta-analysis of data from 170,000 participants in 26 randomised trials. Lancet 2010; 376:1670-1681.

[5] Baigent C, Keech A, Kearney PM et al. Efficacy and safety of cholesterol-lowering treatment: prospective meta-analysis of data from 90,056 participants in 14 randomised trials of statins. Lancet 2005; 366:12671278.

[6] Mihaylova B, Emberson J, Blackwell L et al. The effects of lowering LDL cholesterol with statin therapy in people at low risk of vascular disease: meta-analysis of individual data from 27 randomised trials. Lancet 2012; 380:581-590.

[7] Fulcher J, O'Connell R, Voysey $M$ et al. Efficacy and safety of LDL-lowering therapy among men and women: meta-analysis of individual data from 174,000 participants in 27 randomised trials. Lancet 2015; 385:1397-1405.

[8] Ahn CH, Choi SH. New drugs for treating dyslipidemia: beyond statins. Diabetes Metab J 2015; 39:87-94.

[9] Fruchart JC, Davignon J, Hermans MP et al. Residual macrovascular risk in 2013: what have we learned? Cardiovasc Diabetol 2014; 13:26.

[10] Nordestgaard BG. Triglyceride-Rich Lipoproteins and Atherosclerotic Cardiovascular Disease: New Insights From Epidemiology, Genetics, and Biology. Circ Res 2016; 118:547-563.

[11] Xiao C, Dash S, Morgantini C et al. Pharmacological targeting of the atherogenic dyslipidemia complex: the next frontier in CVD prevention beyond lowering LDL cholesterol. Diabetes 2016; 65:1767-1778.

[12] McBride PE. Triglycerides and risk for coronary heart disease. Jama 2007; 298:336-338.

[13] Kannel WB, Vasan RS. Triglycerides as vascular risk factors: new epidemiologic insights. Curr Opin Cardiol 2009; 24:345-350.

[14] Goldberg IJ, Eckel RH, McPherson R. Triglycerides and heart disease: still a hypothesis? Arterioscler Thromb Vasc Biol 2011; 31:1716-1725.

[15] Chapman MJ, Ginsberg HN, Amarenco P et al. Triglyceride-rich lipoproteins and high-density lipoprotein cholesterol in patients at high risk of cardiovascular disease: evidence and guidance for management. Eur Heart J 2011; 32:1345-1361.

[16] Varbo A, Benn M, Tybjaerg-Hansen A et al. Remnant cholesterol as a causal risk factor for ischemic heart disease. Journal of the American College of Cardiology 2013; 61:427-436.

[17] Jorgensen $A B$, Frikke-Schmidt R, Nordestgaard BG, Tybjaerg-Hansen A. Loss-of-function mutations in APOC3 and risk of ischemic vascular disease. N Engl J Med 2014; 371:32-41.

[18] Nicholls SJ, Brandrup-Wognsen G, Palmer M, Barter PJ. Meta-analysis of comparative efficacy of increasing dose of Atorvastatin versus Rosuvastatin versus Simvastatin on lowering levels of atherogenic lipids (from VOYAGER). The American journal of cardiology 2010; 105:69-76.

[19] Karlson BW, Palmer MK, Nicholls SJ et al. A VOYAGER Meta-Analysis of the Impact of Statin Therapy on Low-Density Lipoprotein Cholesterol and Triglyceride Levels in Patients With Hypertriglyceridemia. The American journal of cardiology 2016; 117:1444-1448.

*This meta-analysis showed that, in patients with high baseline TG levels, LDL-C redction is dependent on the choice and dose of statin, and that TG reduction was less than LDL-C reduction, suggesting the need of additional TG-lowering therapies to reduce the CV risk of these patients 
[20] Barter PJ, Brandrup-Wognsen G, Palmer MK, Nicholls SJ. Effect of statins on HDL-C: a complex process unrelated to changes in LDL-C: analysis of the VOYAGER Database. J Lipid Res 2010; 51:1546-1553.

[21] McTaggart F, Jones P. Effects of statins on high-density lipoproteins: a potential contribution to cardiovascular benefit. Cardiovasc Drugs Ther 2008; 22:321-338.

[22] Corrao G, Conti V, Merlino L et al. Results of a retrospective database analysis of adherence to statin therapy and risk of nonfatal ischemic heart disease in daily clinical practice in Italy. Clinical therapeutics 2010; 32:300-310.

[23] Stroes ES, Thompson PD, Corsini A et al. Statin-associated muscle symptoms: impact on statin therapyEuropean Atherosclerosis Society Consensus Panel Statement on Assessment, Aetiology and Management. Eur Heart J 2015; 36:1012-1022.

[24] Collins R, Reith C, Emberson J et al. Interpretation of the evidence for the efficacy and safety of statin therapy. Lancet 2016; 388:2532-2561.

[25] Gupta A, Thompson D, Whitehouse A et al. Adverse events associated with unblinded, but not with blinded, statin therapy in the Anglo-Scandinavian Cardiac Outcomes Trial-Lipid-Lowering Arm (ASCOT-LLA): a randomised double-blind placebo-controlled trial and its non-randomised non-blind extension phase. Lancet 2017.

*A recent analysis showing that muscle-related event rate report is increased when patients and physicians are aware of the use of statin therapy compared with its blinded use

[26] Bays HE, Davidson M, Jones MR, Abby SL. Effects of colesevelam hydrochloride on low-density lipoprotein cholesterol and high-sensitivity C-reactive protein when added to statins in patients with hypercholesterolemia. The American journal of cardiology 2006; 97:1198-1205.

[27] The Lipid Research Clinics Coronary Primary Prevention Trial results. II. The relationship of reduction in incidence of coronary heart disease to cholesterol lowering. Jama 1984; 251:365-374.

[28] The Lipid Research Clinics Coronary Primary Prevention Trial results. I. Reduction in incidence of coronary heart disease. Jama 1984; 251:351-364.

[29] Jones MR, Nwose OM. Role of colesevelam in combination lipid-lowering therapy. Am J Cardiovasc Drugs 2013; 13:315-323.

[30] Pirillo A, Catapano AL, Norata GD. Niemann-Pick C1-Like 1 (NPC1L1) Inhibition and Cardiovascular Diseases. Curr Med Chem 2016; 23:983-999.

[31] Stitziel NO, Won HH, Morrison AC et al. Inactivating mutations in NPC1L1 and protection from coronary heart disease. N Engl J Med 2014; 371:2072-2082.

[32] Morrone D, Weintraub WS, Toth PP et al. Lipid-altering efficacy of ezetimibe plus statin and statin monotherapy and identification of factors associated with treatment response: a pooled analysis of over 21,000 subjects from 27 clinical trials. Atherosclerosis 2012; 223:251-261.

[33] Derosa G, D'Angelo A, Franzetti IG et al. Efficacy and safety of ezetimibe/simvastatin association on non-diabetic and diabetic patients with polygenic hypercholesterolemia or combined hyperlipidemia and previously intolerant to standard statin treatment. J Clin Pharm Ther 2009; 34:267-276.

[34] Cannon CP, Blazing MA, Giugliano RP et al. Ezetimibe Added to Statin Therapy after Acute Coronary Syndromes. N Engl J Med 2015; 372:2387-2397.

[35] Baigent C, Landray MJ, Reith C et al. The effects of lowering LDL cholesterol with simvastatin plus ezetimibe in patients with chronic kidney disease (Study of Heart and Renal Protection): a randomised placebo-controlled trial. Lancet 2011; 377:2181-2192.

[36] Rossebo $A B$, Pedersen TR, Boman $\mathrm{K}$ et al. Intensive lipid lowering with simvastatin and ezetimibe in aortic stenosis. N Engl J Med 2008; 359:1343-1356.

[37] Ferreira AM, Marques da Silva P. Defining the Place of Ezetimibe/Atorvastatin in the Management of Hyperlipidemia. Am J Cardiovasc Drugs 2017; 17:169-181.

*This review discuss the rationale for the use of the combination atorvastatin-ezetimibe based on the current clinical evidence

[38] Yang YJ, Lee SH, Kim BS et al. Combination Therapy of Rosuvastatin and Ezetimibe in Patients with High Cardiovascular Risk. Clinical therapeutics 2017; 39:107-117.

*This study evalutaed the efficacy and tolerability of rosuvastatin/ezetimibe combination therapy in patients with high CV risk and reported a better efficacy compared with rosuvastatin alone 
[39] Sakamoto K, Kawamura M, Watanabe $T$ et al. Effect of ezetimibe add-on therapy over 52 weeks extension analysis of prospective randomized trial (RESEARCH study) in type 2 diabetes subjects. Lipids Health Dis 2017; 16:122.

[40] Kim KJ, Kim SH, Yoon YW et al. Effect of fixed-dose combinations of ezetimibe plus rosuvastatin in patients with primary hypercholesterolemia: MRS-ROZE (Multicenter Randomized Study of ROsuvastatin and eZEtimibe). Cardiovascular therapeutics 2016; 34:371-382.

[41] Abifadel $M$, Varret $M$, Rabes JP et al. Mutations in PCSK9 cause autosomal dominant hypercholesterolemia. Nat Genet 2003; 34:154-156.

[42] Norata GD, Tibolla G, Catapano AL. Targeting PCSK9 for hypercholesterolemia. Annu Rev Pharmacol Toxicol 2014; 54:273-293.

[43] Sullivan D, Olsson AG, Scott R et al. Effect of a monoclonal antibody to PCSK9 on low-density lipoprotein cholesterol levels in statin-intolerant patients: the GAUSS randomized trial. Jama 2012; 308:2497-2506.

[44] Stroes E, Colquhoun D, Sullivan D et al. Anti-PCSK9 antibody effectively lowers cholesterol in patients with statin intolerance: the GAUSS-2 randomized, placebo-controlled phase 3 clinical trial of evolocumab. Journal of the American College of Cardiology 2014; 63:2541-2548.

[45] Nissen SE, Stroes E, Dent-Acosta RE et al. Efficacy and Tolerability of Evolocumab vs Ezetimibe in Patients With Muscle-Related Statin Intolerance: The GAUSS-3 Randomized Clinical Trial. Jama 2016; 315:1580-1590.

*This trial compares the efficacy of evolocumab or ezetimibe in statin-intolerant patients; interestingly, this study incorporates a novel atorvastatin-controlled, double-blind, crossover phase to identify statin intolerance

[46] Moriarty PM, Thompson PD, Cannon CP et al. Efficacy and safety of alirocumab vs ezetimibe in statinintolerant patients, with a statin rechallenge arm: The ODYSSEY ALTERNATIVE randomized trial. J Clin Lipidol 2015; 9:758-769.

[47] Sabatine MS, Giugliano RP, Keech AC et al. Evolocumab and Clinical Outcomes in Patients with Cardiovascular Disease. N Engl J Med 2017; doi: 10.1056/NEJMoa1615664.

${ }^{* *}$ A clinical trial showing that patient receiving evolocumab had a greater reduction of LDL-C compared with placebo, which translated in a significantly reduced risk of a composite of CV death, myocardial infarction, stroke, unstable angina or coronary revascularization

[48] Catapano AL, Graham I, De Backer G et al. 2016 ESC/EAS Guidelines for the Management of Dyslipidaemias: The Task Force for the Management of Dyslipidaemias of the European Society of Cardiology (ESC) and European Atherosclerosis Society (EAS) Developed with the special contribution of the European Assocciation for Cardiovascular Prevention \& Rehabilitation (EACPR). Atherosclerosis 2016; 253:281-344.

**The most recent guidelines for the management of dyslipidaemias

[49] Perez de Isla L, Alonso R, Watts GF et al. Attainment of LDL-Cholesterol Treatment Goals in Patients With Familial Hypercholesterolemia: 5-Year SAFEHEART Registry Follow-Up. Journal of the American College of Cardiology 2016; 67:1278-1285.

*This registry aimed at evaluating the achievement of LDL-C treatment goals in FH patients and showed that despite the use of intensified lipid-lowering therpaies, most FH patiens still experience high LDL-C levels

[50] Pisciotta L, Fasano T, Bellocchio A et al. Effect of ezetimibe coadministered with statins in genotypeconfirmed heterozygous FH patients. Atherosclerosis 2007; 194:e116-122.

[51] Gagne C, Gaudet D, Bruckert E. Efficacy and safety of ezetimibe coadministered with atorvastatin or simvastatin in patients with homozygous familial hypercholesterolemia. Circulation 2002; 105:2469-2475.

[52] Kastelein JJ, Akdim F, Stroes ES et al. Simvastatin with or without ezetimibe in familial hypercholesterolemia. N Engl J Med 2008; 358:1431-1443.

[53] Kastelein JJ, Ginsberg HN, Langslet G et al. ODYSSEY FH I and FH II: 78 week results with alirocumab treatment in 735 patients with heterozygous familial hypercholesterolaemia. Eur Heart J 2015; 36:29963003.

[54] Ginsberg HN, Rader DJ, Raal FJ et al. Efficacy and Safety of Alirocumab in Patients with Heterozygous Familial Hypercholesterolemia and LDL-C of 160 mg/dl or Higher. Cardiovasc Drugs Ther 2016; 30:473-483. 
[55] Stein EA, Gipe D, Bergeron J et al. Effect of a monoclonal antibody to PCSK9, REGN727/SAR236553, to reduce low-density lipoprotein cholesterol in patients with heterozygous familial hypercholesterolaemia on stable statin dose with or without ezetimibe therapy: a phase 2 randomised controlled trial. Lancet 2012; 380:29-36.

[56] Teramoto T, Kobayashi M, Tasaki H et al. Efficacy and Safety of Alirocumab in Japanese Patients With Heterozygous Familial Hypercholesterolemia or at High Cardiovascular Risk With Hypercholesterolemia Not Adequately Controlled With Statins- ODYSSEY JAPAN Randomized Controlled Trial. Circ J 2016; 80:19801987.

[57] Moriarty PM, Parhofer KG, Babirak SP et al. Alirocumab in patients with heterozygous familial hypercholesterolaemia undergoing lipoprotein apheresis: the ODYSSEY ESCAPE trial. Eur Heart J 2016; 37:3588-3595.

*This trial evaluated the effect of alirocumab in HeFH patients undergoing apheresis and showed a high percentage of apheresis discontinuation in patients treated with PCSK9 inhibitor compared with patients receiving placebo

[58] Raal F, Scott R, Somaratne R et al. Low-density lipoprotein cholesterol-lowering effects of AMG 145, a monoclonal antibody to proprotein convertase subtilisin/kexin type 9 serine protease in patients with heterozygous familial hypercholesterolemia: the Reduction of LDL-C with PCSK9 Inhibition in Heterozygous Familial Hypercholesterolemia Disorder (RUTHERFORD) randomized trial. Circulation 2012; 126:2408-2417.

[59] Raal FJ, Stein EA, Dufour R et al. PCSK9 inhibition with evolocumab (AMG 145) in heterozygous familial hypercholesterolaemia (RUTHERFORD-2): a randomised, double-blind, placebo-controlled trial. Lancet 2015; 385:331-340.

[60] Kastelein JJ, Hovingh GK, Langslet G et al. Efficacy and safety of the proprotein convertase subtilisin/kexin type 9 monoclonal antibody alirocumab vs placebo in patients with heterozygous familial hypercholesterolemia. J Clin Lipidol 2017; 11:195-203 e194.

**In this trial, alirocumab was tested in heterozygous FH patients and showed a great ability to reduce LDLC; with additional beneficial effects also in other class of lipids

[61] Giugliano RP, Pedersen TR, Park J et al. Clinical efficacy and safety of achieving very low LDL-cholesterol concentrations with the PCSK9 inhibitor evolocumab: a prespecified secondary analysis of the FOURIER trial. Lancet 2017; https://doi-org.pros.lib.unimi.it:2050/10.1016/S0140-6736(17)32290-0 .

**This secondary analysis of the FOURIER trial shows a reduction of CV risk as achieved LDL-C level decreased, even down to the first percentile

[62] Nicholls SJ, Puri R, Anderson T et al. Effect of Evolocumab on Progression of Coronary Disease in StatinTreated Patients: The GLAGOV Randomized Clinical Trial. Jama 2016; 316:2373-2384.

${ }^{* *}$ This clinical trial showed the efficacy of evolocumab in reducing the percent atheroma volume in statintreated patients compared with placebo

[63] Kereiakes DJ, Robinson JG, Cannon CP et al. Efficacy and safety of the proprotein convertase subtilisin/kexin type 9 inhibitor alirocumab among high cardiovascular risk patients on maximally tolerated statin therapy: The ODYSSEY COMBO I study. Am Heart J 2015; 169:906-915 e913.

[64] Cannon CP, Cariou B, Blom D et al. Efficacy and safety of alirocumab in high cardiovascular risk patients with inadequately controlled hypercholesterolaemia on maximally tolerated doses of statins: the ODYSSEY COMBO II randomized controlled trial. Eur Heart J 2015; 36:1186-1194.

[65] Maki KC, Guyton JR, Orringer CE et al. Triglyceride-lowering therapies reduce cardiovascular disease event risk in subjects with hypertriglyceridemia. J Clin Lipidol 2016; 10:905-914.

*A meta-analysis suggesting that drugs able to reduce substantially but not exclusively TG and TG-rich lipoproteins may reduce the CV risk of patient with high TG, particularly in those with low HDL-C levels

[66] Jones PH, Nair R, Thakker KM. Prevalence of dyslipidemia and lipid goal attainment in statin-treated subjects from 3 data sources: a retrospective analysis. J Am Heart Assoc 2012; 1:e001800.

[67] Virani SS, Woodard LD, Landrum CR et al. Institutional, provider, and patient correlates of low-density lipoprotein and non-high-density lipoprotein cholesterol goal attainment according to the Adult Treatment Panel III guidelines. Am Heart J 2011; 161:1140-1146.

[68] Keene D, Price C, Shun-Shin MJ, Francis DP. Effect on cardiovascular risk of high density lipoprotein targeted drug treatments niacin, fibrates, and CETP inhibitors: meta-analysis of randomised controlled trials including 117,411 patients. Bmj 2014; 349:g4379. 
[69] Ip CK, Jin DM, Gao JJ et al. Effects of add-on lipid-modifying therapy on top of background statin treatment on major cardiovascular events: A meta-analysis of randomized controlled trials. Int J Cardiol 2015; 191:138-148.

[70] Lee M, Saver JL, Towfighi A et al. Efficacy of fibrates for cardiovascular risk reduction in persons with atherogenic dyslipidemia: a meta-analysis. Atherosclerosis 2011; 217:492-498.

[71] Jun M, Foote C, Lv J et al. Effects of fibrates on cardiovascular outcomes: a systematic review and meta-analysis. Lancet 2010; 375:1875-1884.

[72] Bruckert E, Labreuche J, Deplanque D et al. Fibrates effect on cardiovascular risk is greater in patients with high triglyceride levels or atherogenic dyslipidemia profile: a systematic review and meta-analysis. Journal of cardiovascular pharmacology 2011; 57:267-272.

[73] Pirillo A, Catapano AL. Omega-3 polyunsaturated fatty acids in the treatment of hypertriglyceridaemia. Int J Cardiol 2013; 170:S16-20.

[74] Yokoyama $\mathrm{M}$, Origasa $\mathrm{H}$, Matsuzaki $\mathrm{M}$ et al. Effects of eicosapentaenoic acid on major coronary events in hypercholesterolaemic patients (JELIS): a randomised open-label, blinded endpoint analysis. Lancet 2007; 369:1090-1098.

[75] Marchioli R, Barzi F, Bomba E et al. Early protection against sudden death by $\mathrm{n}-3$ polyunsaturated fatty acids after myocardial infarction: time-course analysis of the results of the Gruppo Italiano per lo Studio della Sopravvivenza nell'Infarto Miocardico (GISSI)-Prevenzione. Circulation 2002; 105:1897-1903.

[76] Ito MK. A Comparative Overview of Prescription Omega-3 Fatty Acid Products. P \& T : a peer-reviewed journal for formulary management 2015; 40:826-857.

[77] Kotwal S, Jun M, Sullivan D et al. Omega 3 Fatty acids and cardiovascular outcomes: systematic review and meta-analysis. Circulation. Cardiovascular quality and outcomes 2012; 5:808-818. 
MS No. MOL280608

STRATEGIES FOR THE USE OF NON-STATIN THERAPIES

Current Opinion in Lipidology

\section{$\underline{\text { Reviewers' comments: }}$}

Excellent overview, containing all relevant information

Some suggestions

1. overall: please perform one more thorough spell and grammar check:

Examples: pg 3: ipolipemic should read: hypolipemic; pg 6: a little effect should preferably read a small impact on etc

We performed spelling and grammar check; the corrections are now highlighted in the text

2. page 9: Just prior to topic 3 (hyperTG), consider to add the reference to the publication which will be online next week August 28th) in Lancet, describing benefit of LDLc reduction in Fourier to levels $<0.2 \mathrm{mmol} / \mathrm{l}$ ! That makes sense to add a short sentence at the end of topic 2 , in order to emphasize the relevance of extremely low LDLc in very high risk subjects? Just a suggestion! (first author: Giugliano)

Thank you for this suggestion. The indicated paper has been discussed in the text and added as reference

3. page 11: before getting into the topic of severe HTG, consider to add 1 sentence reading that this does not include the extreme HTGs (>10 mmol/l?): for these patients, usually with monogenetic severe causes and severely elevated pancreatitis risk, other modalities should be considered (reference to eg. apoCIIIas paper?). Severe range for this topic: 3-10 $\mathrm{mmol} / \mathrm{l}$ ?

As suggested, in the indicated topic we added a sentence specifying that we only considered mild-to-moderate HTG patients and excluded severe HTG from the discussion as it was beyond the purpose of this review 